\title{
LMPS: Localized Multi-path Selection for QoS Routing in VoIP Networks
}

\author{
Khaled M.F. Elsayed, Hassan Fadel, and Amin M. Nassar
}

\author{
Department of Electronics and Communications Engineering \\ Faculty of Engineering, Cairo University, Giza, Egypt 12613 \\ khaled@ieee.org, hassan.fadel@egti.com.eg, anassar@eng.cu.edu.eg
}

\begin{abstract}
Localized QoS routing techniques were proposed to achieve acceptable performance without exchanging global state information over the network. In such techniques, the ingress nodes estimate the network state and make the routing decision locally, hence reducing the signaling traffic at core routers. However, using only locally collected information to make routing decision is not a completely reliable technique to achieve trusted QoS performance. The Localized Multi-Path Selection (LMPS) scheme is proposed in this paper for connection-oriented bandwidth-aware flows to overcome the common drawbacks of the localized techniques. The LMPS technique is a multi-path selection algorithm, which selects paths that are capable of satisfying the requested bandwidth requirement of an incoming call, while at the same time trying to avoid the overloaded links to minimize the overall blocking ratio in the network. We demonstrate through extensive simulations and comparisons with a fully localized approach known as virtual capacity routing (VCR) and a shortest-widest multipath scheme based on QOSPF that the LMPS scheme provides good performance.
\end{abstract}

\section{Introduction}

Most QoS routing approaches require network routers to periodically exchange link state information in order to obtain a global view of the network state. As network resource availability changes continuously with traffic dynamics, maintaining accurate network state information for provisioning of acceptable service quality requires frequent information exchange in the network. The fast change in network state and the associated regular state updates could lead to large amount of signaling traffic especially in large scale networks. Furthermore, the core routers, whose main function is to forward packets as fast as possible, could be overburdened by tasks such as collecting and exchanging the information that is usually needed to perform qualityof-service $(\mathrm{QoS})$ routing. Also, the network QoS state information maintained by the network nodes can become out-of-date if the QoS state update interval is large rela- 
tive to the time scale of traffic dynamics. Furthermore, path selection based on shortest path algorithms such as Dijkstra's algorithm does not seem to be well-justified in the presence of expired state information. Another well-known problem is path oscillations which may occur when a node discovers that a certain link is congested and after the next route selection will avoid routing any traffic on these links resulting in their under-utilization. The links then seem attractive again and re-selected by the routing algorithm. This oscillatory behavior can have a negative impact on the system performance.

As an alternative to the global QoS routing schemes, localized approaches $[2,7,8,10]$ were introduced to maintain QoS routing without exchanging global state information. In these approaches, nodes infer the network QoS state using locally collected information based on flow blocking statistics, and perform flow routing using this localized view of the network QoS state. The localized QoS routing approaches have several advantages. The communication overhead involved is minimal, with no global information exchange. Also the core routers are not involved with interpreting QoS signaling messages and do not need to keep or update any QoS state database necessary for global QoS routing.

Nelakuditi, and Zhang [2] proposed an adaptive proportional QoS routing method which makes local routing decisions based on locally collected QoS state information (with no global network information exchange among routers). They compared the performance of these localized routing methods with that of a global optimal proportional model that has knowledge of the global network QoS state. They demonstrated that an appropriately designed localized QoS routing scheme that makes routing decisions solely based on local information can potentially achieve such global objectives as a stable network system with good overall system performance.

Extensions to support QoS routing based on OSPF have been proposed in the QOSPF [1] protocol. The QOSPF is considered as an extension to OSPF to support QoS by flooding the network with information about the available and used link resources. In this proposal the routing decisions are being done based on topology, link resources available and traffic requirements. The QOSPF framework signals ingress routers to send the QoS requirements for incoming traffic. If a QoS route can be computed and a path reserved, a message is sent back, reserving the resource and accepting the request. In [3] Lakkakorpi describes the extensions suggested to the OSPF protocol to support QoS routes. An overview for QoS routing and examples of source routing protocols are discussed in [4]. More information about QoS extension to OSPF can be found in [1], [5] and [6]

In this paper, we propose the Localized Multi-Paths Selection (LMPS) technique to overcome the common drawbacks of the localized approaches like the inflexibility in bandwidth assignment on some paths and the inaccurate traffic parameters calculations. We apply LMPS to VoIP networks where all VoIP calls have the same bandwidth. Information about the used paths are exchanged between the ingress/egress nodes without involving the core routers. We can classify LMPS to lie somewhere in between the global link-state QoS schemes and the localized fully protocols. The simulations indicate that the LMPS is capable of achieving good performance compared to the mentioned localized protocols. 


\section{System Model}

We consider a carrier network for transporting of VoIP calls as depicted in Fig. 1. Ingress/egress IP routers (abbreviated as IE nodes or routers) are connected to a traditional PSTN-based local exchanges and/or PBXs. Core routers are used to interconnect the IE routers. The IE routers contain the necessary functionality to process the PSTN signaling and format conversion. All PSTN calls can be optionally transcoded at the IE nodes for bandwidth reduction.

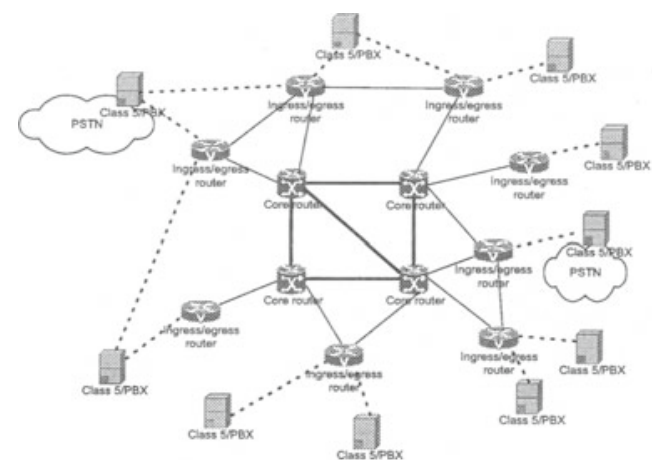

Fig. 1. The system model

A link-state protocol such as OSPF is employed within the network. It is used to collect information about network state and topology updates. All techniques discussed here use the information collected by the link-state protocol to select candidate paths.

The offered load for a generic source-destination (SD) IE node pair is given by $\mathrm{V}$ $=\lambda \times \mathrm{AHT}$, where $\lambda$ presents the call arrival rate (calls/sec) for the SD pair, and AHT is the average holding time for the calls. Let the capacity of a generic link be $C_{l}$ bits/sec and let $B$ be the required bandwidth for (an optionally transcoded) phone call in bits/sec.

The objective is to route calls for each SD pair in the most efficient and scalable manner possible. More precisely, it is required to reduce the blocking probability for establishing a new call and to maximize the potential revenue and network utilization. While such a network can be used in a multiservice setting where VoIP calls are mixed with other traffic types such as best-effort data-oriented traffic, out concentration will be a VoIP dedicated network or where a fixed portion of bandwidth is allocated for VoIP calls. 


\section{Localized Quality of Service Routing Approaches}

The localized QoS routing techniques of $[2,8]$ were developed based on the concept of the "virtual capacity". This concept takes into account that typically the paths between an SD pair could traverse links shared with other SD pairs. A bottleneck link of a path is defined as the link offering the path the minimum (virtual) capacity. Furthermore, as traffic patterns across a network change, the bottleneck link of a path and its capacity could also change. The virtual capacity is a function of the offered load to the links belonging to the path and the perceived blocking probability at the IE node. Assume that a path $r$ between an SD pair carries offered load $v_{r}$, and the corresponding blocking probability observed by the IE node is $b_{r}$. The blocking probability on this path can be presented using the Erlang's loss formula as function of both the path load $v_{r}$ and the capacity $c_{r}$ as follows:

$$
b_{r}=E\left(v_{r}, c_{r}\right)
$$

If the quantities $v_{r}$ and $b_{r}$ are measured, an estimate of the virtual capacity of the path $v c_{r}$ can be obtained by the inverse of the Erlang function:

$$
v c_{r}=E^{-1}\left(v_{r}, b_{r}\right)
$$

If $m$ paths $\left(r_{1}, r_{2}, \ldots, r_{i}, \ldots, r_{m}\right)$ share a link with capacity $c$, then the virtual capacity $v c_{i}$ of path $r_{i}$ represents its "capacity share" of the link. Let $v_{i}$ denote the offered load on the link from path $r_{i}$. The blocking probability on the link is given by $b=E\left(\sum_{i=1}^{m} v_{i}, c\right)$. In the case when all flows have the same bandwidth requirement, and the equalizing of the blocking probability for all paths sharing a given link is a target, the virtual capacity of path $r_{i}$ is given by:

$$
v c_{i}=E^{-1}\left(v_{i}, b_{i}\right)=E^{-1}\left(v_{i}, b\right)
$$

where, $b_{i}$ denotes the observed blocking probability of path $r_{i}$. We can see from (3) that for path $r_{i}$ the larger the offered load $v_{i}$ is, the larger is its virtual capacity $v c_{i}$.

The virtual capacity routing (VCR) model $[2,8]$ is based on load balancing across multiple paths (it is to be noted that the selected paths are fixed). Initially, a set of paths are identified using a route selection scheme such as shortest-hop algorithm. In the VCR scheme, the IE nodes estimate a certain virtual capacity for each path as a private share in the shared links traversed by the path. Let the overall offered traffic to SD pair $(s, d)$ be $V$, and let there be $m$ available paths between (s,d), then the load balancing problem is finding the set of flow proportions $\left\{\alpha_{i}\right\}_{i=1}^{m}$, with the constraint $\sum_{i=1}^{m} \alpha_{i}=1$. The offered load to path $i$ is then given by $\alpha_{i} V$. The values $\left\{\alpha_{i}\right\}_{i=1}^{m}$ are dynamically updated to shift traffic away from paths observing high blocking. The algorithm uses the equalizing blocking probability (EBP) strategy to simplify and solve the minimization problem of the blocking probability of the paths. 
The VCR algorithm depends on an observation period to measure the quantities of interest. The observation period is divided into two intervals. In the first interval (blocking observation interval) the IE nodes collect local statistics regarding the blocking on each path, to be able to adapt its traffic parameters to minimize the blocking probability on this path. The adaptation is being done during the second interval (traffic parameters recalculation interval), where each IE node recalculates some traffic parameters to be able to minimize the blocking probability on this path during the next cycle. More details about QoS routing without exchanging global information can be found in [7], and about the VCR model in [2,8].

\section{The Localized Multipath Selection (LMPS) Scheme}

One of the drawbacks of localized techniques of [2,8] is that the set of multiple paths is fixed. If the bottleneck links of the paths are overloaded, then there is not much that could be done to reduce the blocking as shifting traffic may also lead to a path with high blocking probability. Another drawback is the possibility of reaching inaccurate decisions due to errors in the blocking observation and load proportions calculations.

The LMPS technique is based on identifying the currently best possible set of paths satisfying the bandwidth and other QoS constraints to be used by each SD pair, in order to minimize blocking probability on each path. In LMPS, network state information is collected and exchanged only by the IE nodes on an on-demand basis when replacement of current paths is needed. This is done in conjunction with the localized approach. This hybrid of global and local information helps to compensate the drawbacks of the traditional localized technique and lead to better QoS performance without the common drawbacks of the global state protocols (such as QOSPF).

The observation mechanism: The observation process in the LMPS technique depends on the observation period $T_{g}$. The period $T_{g}$ is the time interval between two successive instants at which a node examines its paths. The period $T_{g}$ consists of two intervals: the path checking interval $T_{p c}$ recalculation interval $T_{r c}$. During and period $T_{p c}$ each node checks the status of its paths, and may decide to create new ones. It then sends updates to all other IE nodes regarding its new set of paths. The other nodes update their conceived network state according to the updates. The above process occurs once during $T_{g}$ (within $T_{p c}$ ) at any time, and it does not matter if every node executes it in different time therefore storms of signaling traffic are not likely to occur. The second interval is the traffic parameters recalculation interval $T_{r c}$ where all IE nodes are required to start the second time interval at approximately the same time, and exit it also at the same time. The interval $T_{r c}$ is relatively small (few seconds), and within it each IE node recalculates its new paths parameters according to the new set of paths selected by each IE node and the observed blocking of its paths. The path updates tables will be discussed later in details.

The LMPS algorithm: Each IE node picks $m$ (typically $m=3$ ) paths satisfying the fixed QoS constraints (propagation delay, reliability, ..) to each destination from the network topology information collected by the link-state protocol. Each IE node broadcasts its own set of paths and their respective links to other IE nodes. An IE 
node also keeps a table of its own paths and the paths of other IE nodes. Using this information, each IE node can identify how many paths share a specific link and then determines its own capacity share in each physical link, and the most shared link (bottleneck link) in each path. If the number of paths using link $l$ is given by $N_{l}$, then the link with the smallest $\mathrm{C}_{l} / B N_{l}$ is the bottleneck link. Here, the bottleneck is defined as function of number of paths rather than load, since the node exchange the path information with the assumption that links are fairly shared among the paths.

After each IE node determines the bottleneck link(s) of its own paths, it calculates the virtual capacity of each path. The virtual capacity of a path in LMPS is given by the maximum number of calls that can be used by the path and is equal to $C_{l} / B N_{l}$ at the bottleneck link. This is based on the assumption that traffic between all SD pairs is uniform and all calls request identical amount of bandwidth. However, in the case of non-uniform traffic or call bandwidths, the virtual capacity of each path should be weighted by the service provider as part of a service level agreement (SLA). LMPS restricts the number of active calls on any path to be less than or equal to its virtual capacity. In contrast to the VCR approach, we note that there is no need to calculate the virtual capacity using inverse Erlang formula. The IE nodes use the resulting virtual capacity to calculate the related path proportions $\alpha_{i}$, for each path. If we have $m$ active paths with LMPS virtual capacities $\left\{\overline{v C}_{i}\right\}_{i=1}^{m}$, then the flow proportions are simply given by $\alpha_{i}=\overline{v c}_{i} / \sum_{i=1}^{m} \overline{v c}_{i}, i=1, \cdots, m$. The offered load to path $i$ is then $v_{i}=\mathrm{V} \times \alpha_{\mathrm{i}}$, where $V$ is total offered load to a given SD pair. A counter $C_{i}$ records the number of calls routed on path $i$.

At the start of the period $T_{p c}$, each IE node checks for paths satisfying the condition $C_{i} \quad \psi \times v c_{i}$, where $\psi$ is configurable ratio with default value one. However, the ratio $\psi$ may be set to a value less than one (e.g. 0.8 ) as a protective procedure to discover the blocked paths before the blocking occurs. If blocked paths are found, the node marks the bottleneck links of these paths as forbidden links, and selects as many new paths as needed to replace the blocked paths with the constraint of avoiding the forbidden links. After identifying the new set of paths, the nodes with newly created paths broadcast their new paths information to all other IE nodes. In the mean time, the IE nodes receive information from other nodes about their paths. By the end of period $T_{p c}$, all nodes assume no further updates will be broadcast and they start calculating the parameters of the new paths (virtual capacity, flow proportions, flow load, path counter initial value...) to be able to route calls via these new paths. It is so important that all IE nodes start the interval $T_{r c}$ and leave it at the same time (at least with reasonable time differences) to start using the new paths simultaneously. It is very important to note that currently existing calls are kept on whatever paths are chosen and new routes are used by the new incoming calls, i.e. no rerouting in involved. The core routers have no role to play in this process (except forwarding the messages between the IE nodes), thereby simplifying the design of such routers and reducing the processing overhead.

LMPS signaling information: LMPS is based on exchanging some global information between the IE nodes to achieve reliable QoS performance. This global in- 
formation is considered as signaling traffic, and can be classified as: 1) path updates messages which are sent by an IE node to all other IE nodes to announce a new paths set; 2) acknowledgment messages with which an IE node confirms reception of a path update message; 3 ) link state requests: when an IE node changes its paths, it needs to know the current load on each new path, so it sends request to the routers located on the new path to get this information; and 4) link state update: as a response for the (link state request), each node sends an information about single physical link to the requesting IE node.

The amount of signaling information in LMPS (during a fixed period) is a function of factors such as the number of IE nodes in the network, the number of blocked paths reported every $T_{g}$, the average path length in the given topology, and the number of parallel paths used for each SD pair. The OSPF protocol can be tailored to transport the signaling information used by LMPS. However, the signaling traffic in the LMPS technique is not periodic and is only generated when an IE node needs to change its paths.

Time synchronization: In the LMPS technique, IE nodes are required to maintain time synchronization among themselves to be able to proceed in the time interval $T_{r c}$ simultaneously, or at least with acceptable reasonable time differences. The worst case performance occurs when $T_{r c}$ intervals from different IE nodes are completely unsynchronized. This causes an IE node to repeat its traffic parameters calculations several times during the period $T_{g}$ due to the frequent changes. This problem can be solved by using network time synchronization protocol like the Simple Network Time Protocol sNTP [9].

\section{Performance Evaluation}

Since the focus in this paper is routing of VoIP calls, the blocking probability is considered as the main metric to evaluate the performance of the different routing schemes. The Network Simulator NS-2 is used to simulate the mentioned models. NS-2 is a discrete-event packet level multi-protocol simulator.

The voice CODEC G.729 (voice rate $8 \mathrm{~kb} / \mathrm{s}$ voice) is used to generate voice traffic during the simulation. The call bandwidth is $14.8 \mathrm{kbps}$ after using the RTP header compression (cRTP). Two network topologies are used during the simulation. The first one called NSFNET (Fig. 2a), with 14 nodes, 21 links, average node connectivity degree $=3$, and five IE nodes. The nodes $(0,1,2,3$ and 4$)$ are selected to act as IE nodes (the choice is mainly for evaluation purposes not for specific reasons with these nodes). The second topology is called LCTNET (Fig. 2b), with 12 nodes, 13 links, average node connectivity degree $=2.167$, and $4 \mathrm{IE}$ nodes. The nodes $(0,1,2$ and 3$)$ are selected to act as IE nodes. We select the NS-2 class based queuing (CBQ) as the queue management technique, and the weighted round robin (WRR) queuing discipline. A constraint of $250 \mathrm{msec}$ is assumed on the end-to-end delay (including both propagation and processing delays). Three parallel paths are used to route calls for each SD pair. For the two network topologies, we set all links capacities to be equal to two Mbps (unchannelized E1). 
The simulation considers using two levels of call generation rates $\left(\lambda_{1}, \lambda_{2}\right)$. The rate $\lambda_{1}$ presents the normal call arrival rate for most SD pairs in the network, while the rate $\lambda_{2}$ presents the arrival rate for the hot pairs in the network (higher rates). The factor $\beta$ presents the degree of the non-uniformity in the network $\beta=\frac{x \lambda_{2}}{x \lambda_{2}+(1-x) \lambda_{1}}$ where $x$ is the percentage of hot pairs, and $(1-x)$ is the percentage of normal pairs from the total number of SD pairs in the network. The average call arrival rate for each SD pair is presented by $\lambda$ given by: $\lambda=\frac{\lambda_{T}}{n(n-1)}=(1-x) \lambda_{1}+x \lambda_{2}$, where $n$ is number of IE nodes, and $n(n-1)$ is number of SD pairs. $\lambda_{T}$ presents the total rate of call generation in the network (by all IE nodes) every second: $\lambda_{T}=n(n-1)(1-x) \lambda_{1}+n(n-1) x \lambda_{2}$. The rate of the normal pair can be expressed as:

$$
\lambda_{1}=(1-\beta) \lambda_{T} / n(n-1)(1-x)=(1-\beta) \lambda /(1-x)
$$

and of the hot pair as:

$$
\lambda_{2}=\beta \lambda_{T} \cdot / n(n-1) x=\beta \lambda / x
$$

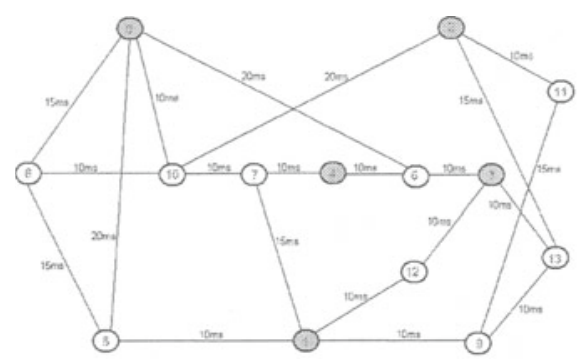

(a)

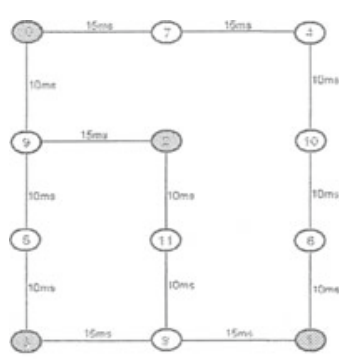

(b)

Fig. 2. The network topologies used in the experiments

The simulation compares three models to evaluate the QoS routing performance. The first model is the LMPS routing algorithm, the second is the pure localized approach multi-path VCR, and the third model is the multipath selection based on SWP and QOSPF protocol signaling (explained below). All algorithms are evaluated for the case of three parallel paths for each SD pair. All models consider two QoS constraints, the bandwidth availability and end-to-end delay restrictions. Paths with delay exceeds $250 \mathrm{msec}$ are rejected. 


\subsection{Multipath Routing with Global QoS Information}

To assess the performance of LMPS against routing protocols with global QoS information, we utilize a multipath routing model with QOSPF-based signaling. The model works by selecting $m$ paths for each SD pair using a shortest-path algorithm. These paths are fixed. When a call arrives, the shortest-widest path (SWP) policy is used to select the best path (from the $m$ paths) to establish the incoming call. The QOSPF updates are sent regularly every $\mathrm{T}_{\text {upd }}$, to report the changes in the capacity status on each link in the network. In QOSPF, there are two types of signaling messages exchanged, the RES-LSA messages, and the Acknowledgment messages. The amount of signaling information in QOSPF (during fixed period) is being affected by some factors like the update period $T_{u p d}$, and the connectivity degree of each node.

\subsection{Performance with Uniform Traffic Loading}

Fig. 3 and Fig. 4 compare the blocking behavior for the three models using the NSFNET and LCTNET topologies respectively. The common parameter is $\beta=0.333$. The graphs show that the LMPS model has better performance than the traditional VCR, and less than the performance of the QOSPF model. The QOSPF-based multipath SWP has the best performance (if used with tight update periods), but costs other disadvantages that were discussed earlier. In case of the LCTNET, the LMPS model has performance closer to the VCR performance. The reason is that the topology of the LCTNET (low node connectivity degree) doesn't offer many alternative paths to recover from blocked paths when they occur.

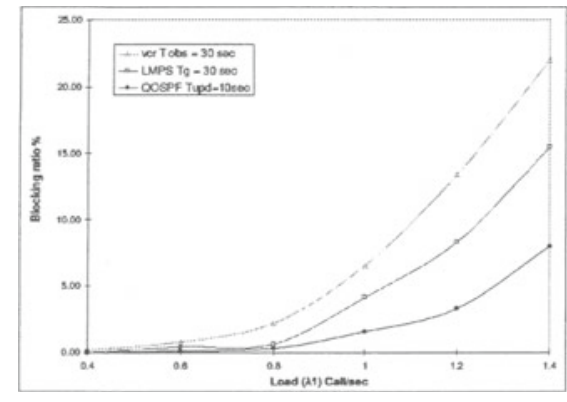

Fig. 3. The blocking behavior (NSFNET)

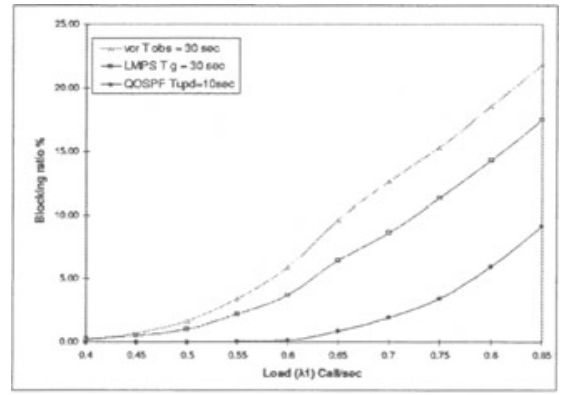

Fig. 4. The blocking behavior (LCTNET)

\subsection{Performance with Non-uniform Traffic Loading}

The factor $\beta$ reflects the non-uniformity of the traffic in the network. As $\beta$ increases, the network suffers from hotspots (paths with hot traffic load $\lambda_{2}$ ), while the total load in the network is always constant. The percentage $x$ (percentage of hot pairs) was configured to be $20 \%$ of the SD pairs in the network (hot pairs with rate $\lambda_{2}$ ), while the 
remaining pairs (80\%) carry normal load with rate $\lambda_{1}$. For each value of $\beta$, the values of $\lambda_{1}$ and $\lambda_{2}$ are adjusted using equations (4) and (5) while keeping the value of the average and total loads respectively $\lambda$ and $\lambda_{\mathrm{T}}$ constant. We set $\lambda=0.96 \mathrm{calls} / \mathrm{sec}$ while changing the value of $\beta$ and show the result in Fig. 5. It shows that changing the traffic uniformity and distribution affects the overall blocking ratio directly. The most affected model as is the VCR model. The QOSPF-based multipath SWP and the LMPS models are more capable of coping with the hot spots occurring on some the links.

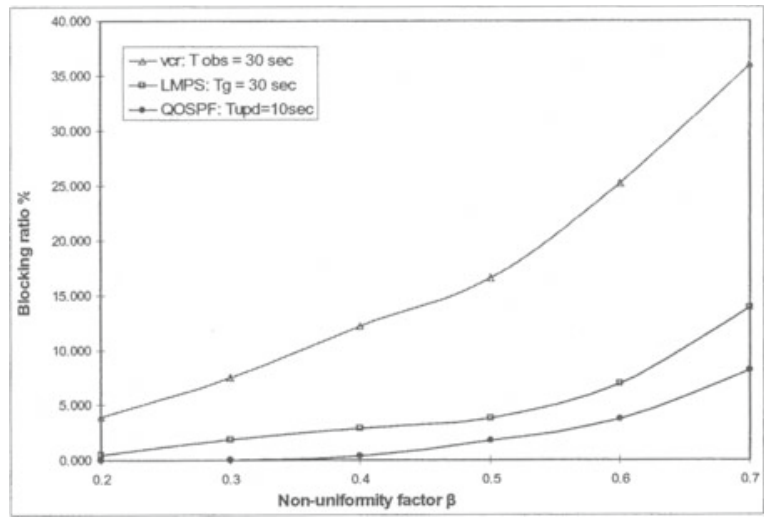

Fig. 5. Effect of changing the non-uniformity factor $\beta$

\subsection{Network Utilization and Link Over-Utilization}

In the utilization calculation, the number of hops used by each call is being considered. The network utilizations for three models are compared using the NSFNET topology with $\beta=0.333$. Fig. 6 shows that the traditional localized proportional approach (VCR) has the highest network utilization values compared to the LMPS and the QOSPF models. As an indication for the algorithm efficiency, the load of the links is monitored during the simulation to record any overflow. The overflow indicates that some calls are accepted on certain physical links although that no physical capacity is available on these links. The over utilization is not accepted in networks with QoS guarantees as this reflect the incapability of the network to provide guaranteed QoS. Fig. 7 shows the maximum overflow for each model during its operation. The overflow load is being monitored against the call arrival rate $\lambda_{1}$, with fixed nonuniform factor $\beta=0.333$ at the LCTNET network with 2Mbps links (135 calls as integer capacity). The dashed horizontal line (at 135 calls) indicates the restriction on each physical link (maximum capacity 135 calls). The relatively long update period $\left(T_{\text {upd }}\right)$ in the QOSPF model result in accepting calls while no capacity available in some links. LMPS (with its capability to change paths) does not allow the existence of the blocked paths, hence reduces the overflow on physical links. 


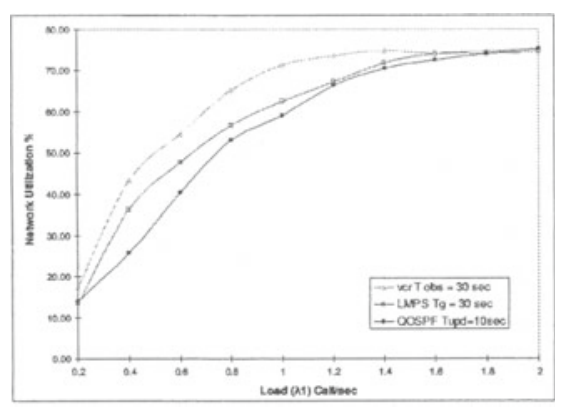

Fig. 6. Network utilization

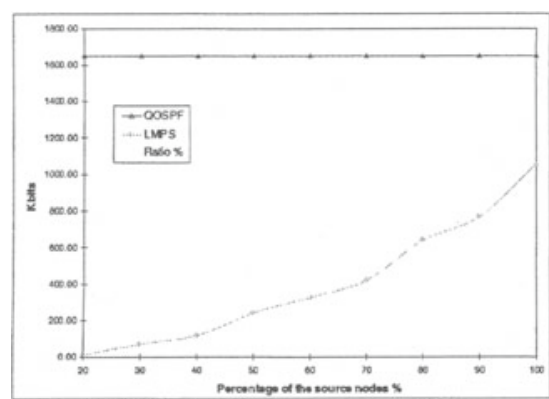

Fig. 8. The effect of changing the percentage of source nodes in the network

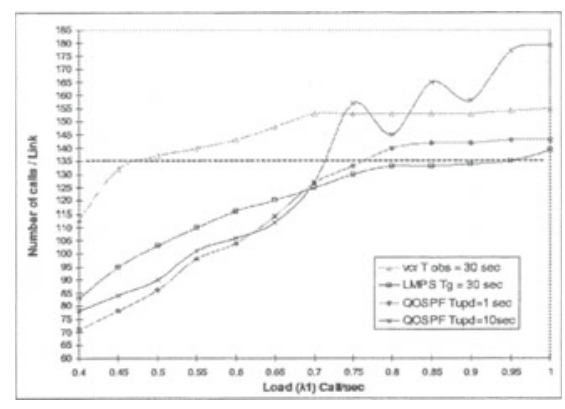

Fig. 7. The link over-utilization

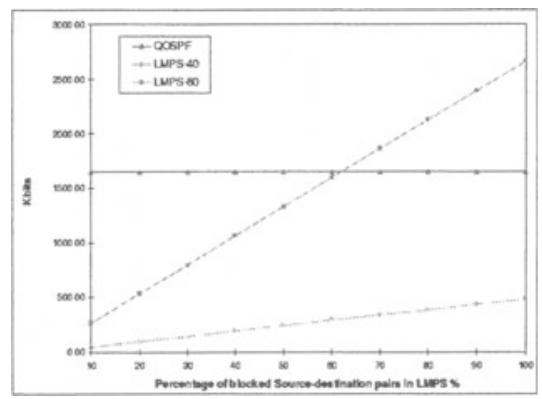

Fig. 9. The effect of changing the percentage of the blocked sourcedestination pairs in LMPS model

\subsection{Signaling Overhead}

The amount of the signaling information exchanged by the nodes in the network reflects the efficiency of the QoS algorithm and its capability to save the network resources. In this section, a comparison between both QOSPF and LMPS algorithms shows the amount of signaling information exchanged by each one. Increasing the percentage of the IE nodes in the network affect the amount of signaling information exchanged by the LMPS model, where the signaling information is being exchanged only between the IE nodes. In QOSPF the signaling information is being exchanged between all nodes, hence increasing the IE nodes in the network does not affect the QOSPF signaling information directly. This is illustrated in Fig. 8.

Another important factor to consider in the LMPS model is the percentage of SD pairs suffering from blocked paths during a single update period. This affects the amount of path updates exchanged by the IE nodes. Fig. 9 shows the effect of changing the percentage of SD pairs with blocked paths. The LMPS-40 and LMPS-80 curves represent the cases when $40 \%$ respectively $80 \%$ of the nodes in the NSFNET are IE nodes. 


\section{Conclusions}

We proposed LMPS as a multipath routing protocol that uses local decisions to load balance traffic across multiple paths. It uses global information that approximates the network state and is only exchanges between the IE nodes and only triggered when saturated paths are identified. The LMPS approach proved superior to fully localized approaches such as (VCR). It should be noticed that the operation of the LMPS approach depends strongly on the availability alternate paths between the IE nodes in the network. Using the LMPS approach in networks with low connectivity (nodes have low connectivity degrees), and as a result a small number of paths between IE nodes exist, is not advisable. In the worst cases, the LMPS will act similar to the traditional localized techniques (e.g. the adaptive proportional approach). The best topologies are when a plethora disjoint paths for each SD pair exist.

Further evidence of LMPS stability and susceptibility to path oscillation is needed. Also, packet-level performance and assessment of typical signaling information overhead should be investigated. Another important area for investigation is effect pf exchanging actual path load between the IE nodes rather in addition to the set of links comprising the path.

\section{References}

1. G. Apostolopoulos et al., RFC 2676 QoS Routing Mechanisms and OSPF Extensions.

2. S. Nelakuditi, Z.-L. Zhang, R.P. Tsang, Adaptive Proportional Routing: A Localized QoS Routing Approach, Proc. of IEEE INFOCOM , pp. 1566-1575, 2000.

3. J. Lakkakorpi, QoS Routing Extensions to OSPF, technical report Helsinki University of Technology, available from http://keskus.hut.fi/tutkimus/ipana/paperit/, 2000.

4. S. Chen and K. Nahrstedt, An Overview of Quality of Service Routing for NextGeneration High-Speed Networks: Problems and Solutions, IEEE Network, Vol. 12, pp. 64 -79, Nov./Dec. 1998.

5. G. Apostolopoulos, R. Guerin and S. Kamat, Implementation and Performance Measurements of QoS Routing Extensions to OSPF, Proc. IEEE INFOCOM, pages 680-688, 1999.

6. Z. Zhang, C. Sanchez, B. Salkewicz, E. Crawley, QoS Extension to OSPF, IETF Internet draft, Sep 1997.

7. S. Nelakuditi, R. P. Tsang, and Z.-L. Zhang, Quality-of-Service Routing without Global Information Exchange, Proc. of IWQOS 1999.

8. S. Nelakuditi, S. Varadarajan, and Z.-L. Zhang, On Localized Control in Quality-ofService Routing, IEEE Transactions on Automatic Control, Systems and control Methods for Communication Networks, June 2002.

9. D. L. Mills, RFC 1769 Simple Network Time Protocol V3.

10. I. Gojmerac, T. Ziegler, P. Reichl: Adaptive Multipath Routing Based on Localized Distribution of Link Load Information. Proc. QofIS'03, Stockholm, Oct. 2003. 